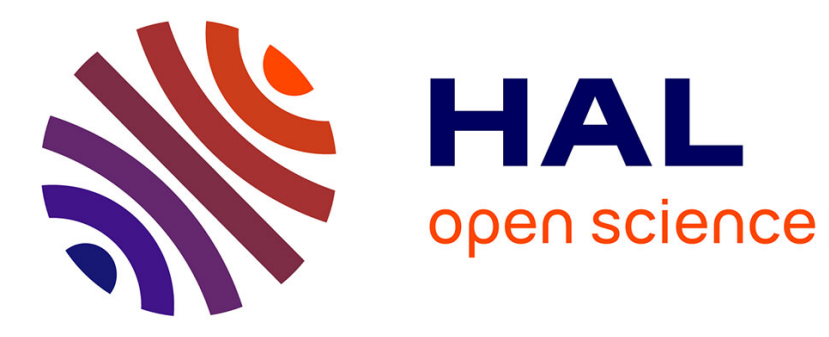

\title{
Coherent Resonant Tunneling through Double Metallic Quantum Well States
}

Bingshan Tao, Caihua Wan, Ping Tang, Jiafeng Feng, Hongxiang Wei, Xiao Wang, Stéphane Andrieu, Hongxin Yang, Mairbek Chshiev, Xavier Devaux, et al.

\section{To cite this version:}

Bingshan Tao, Caihua Wan, Ping Tang, Jiafeng Feng, Hongxiang Wei, et al.. Coherent Resonant Tunneling through Double Metallic Quantum Well States. Nano Letters, 2019, 19 (5), pp.3019-3026. 10.1021/acs.nanolett.9b00205 . hal-02999633

\section{HAL Id: hal-02999633 https://hal.science/hal-02999633}

Submitted on 20 Nov 2020

HAL is a multi-disciplinary open access archive for the deposit and dissemination of scientific research documents, whether they are published or not. The documents may come from teaching and research institutions in France or abroad, or from public or private research centers.
L'archive ouverte pluridisciplinaire HAL, est destinée au dépôt et à la diffusion de documents scientifiques de niveau recherche, publiés ou non, émanant des établissements d'enseignement et de recherche français ou étrangers, des laboratoires publics ou privés. 


\section{Coherent Resonant Tunneling through Double Metallic Quantum-Well States}

Bingshan Tao ${ }^{1,2,3}$, Caihua Wan ${ }^{1}$, Ping Tang ${ }^{1}$, Jiafeng Feng ${ }^{1}$, Hongxiang Wei ${ }^{1}$, Xiao Wang ${ }^{1}$, Stéphane Andrieu $^{2}$, Hongxin Yang ${ }^{4}$, Mairbek Chshiev ${ }^{5}$, Xavier Devaux ${ }^{2}$, Thomas Hauet ${ }^{2}$, François Montaigne ${ }^{2}$, Stéphane Mangin ${ }^{2}$, Michel Hehn², Daniel Lacour ${ }^{2}$, Xiufeng Han ${ }^{1 \dagger}$, and Yuan $\mathrm{Lu}^{2 *}$

${ }^{1}$ Beijing National Laboratory of Condensed Matter Physics, Institute of Physics, University of Chinese Academy of Sciences, Chinese Academy of Sciences, Beijing 100190, China

${ }^{2}$ Université de Lorraine, CNRS, Institut Jean Lamour, UMR 7198, campus ARTEM, 2 Allée André Guinier, 54011 Nancy, France

${ }^{3}$ Institute of Electrical Engineering, Chinese Academy of Sciences, Beijing 100190, China

${ }^{4}$ Key Laboratory of Magnetic Materials and Devices, Ningbo Institute of Materials Technology and Engineering, Chinese Academy of Sciences, Ningbo 315201, China

${ }^{5}$ Univ. Grenoble Alpes, CEA, CNRS, Grenoble INP, INAC-Spintec, 38000 Grenoble, France

Corresponding authors:

*yuan.lu@univ-lorraine.fr

†xfhan@iphy.ac.cn

\section{Abstract}

Study of resonant tunneling through multi-metallic quantum well (QW) structure is not only important for the fundamental understanding of quantum transport, but also for the great potential to generate advanced functionalities of spintronic devices. However, it remains challenging to engineer such structure due to the short electron phase coherence length in metallic QW system. Here, we demonstrate the successful fabrication of double-QW structure in a single fully epitaxial MTJ heterostructure, where two Fe QW layers are sandwiched between three $\mathrm{MgAlO}_{x}$ tunnel barriers. We show clear evidence of the coherent resonant tunneling through the discrete QW states in the two QWs. The coherent resonant tunneling condition is fulfilled only when the middle barrier between the two QWs is thin enough and available QW states are present simultaneously in both QWs under a certain bias. Compared to the single QW structure, the resonant tunneling in double-QW MTJ produces strong conductivity oscillations with much narrower peak width (about half) owing to the enhanced energy filtering effect. This study presents a comprehensive understanding of the resonant 
tunneling mechanism in MTJ with multiple QWs, which is essential for future development of new spintronic devices operating in the quantum tunneling regime.

Keywords: quantum well; magnetic tunnel junction; resonant tunneling; magnetoresistance 


\section{Introduction}

Resonant tunneling in quantum well (QW) structures has been extensively studied because of its importance in the field of nanoelectronic science and technology. ${ }^{1}$ Recently, in metallic QW system, the combination of the tunneling magnetoresistance $(\mathrm{TMR})^{2,3}$ effect with resonant tunneling through metallic QW states in magnetic tunnel junctions (MTJs) has triggered considerable interest in the new functionality of spintronic devices. ${ }^{4-8}$ In these structures, the discrete spin-dependent QW states can be formed in the middle metallic QW layer sandwiched between two barriers. The QW potential barrier can be formed either by metallic layer using the symmetry dependent Bloch state orbital band structure, ${ }^{9-12}$ or by a double oxide tunneling barriers with a much higher barrier height for better electron confinement. ${ }^{13-15}$ Since the QW states are often formed in the majority spin channel, ${ }^{4,8}$ the conductance in parallel magnetic configuration can be enhanced when the injector Fermi level is aligned with the QW state at resonance, resulting in an enhanced TMR ratio. ${ }^{4} \mathrm{~A}$ record high resonant TMR $(\approx 4000 \%)$ has been reported for a double barrier MTJ measured with point-contact technique at $4.2 \mathrm{~K} \cdot{ }^{16}$

In semiconductor materials, multi-QW structures have already been extensively studied, ${ }^{17}$ such as resonant-tunneling diode, ${ }^{18}$ multi-quantum well light emitting diode and laser ${ }^{19}$ etc. When the thickness of the barrier and QW is thin enough, the multi-QWs form the superlattice structure and the QW states in different wells can hybridize with each other to create new sub-band structure, ${ }^{20,21}$ which gives rise to many novel features for electronic and optronic properties. However, up to now, no experimental results have been reported for the metallic multi-QW structures. The general reason is due to the difficulty to keep a good electron phase coherence when resonant tunneling through multi metallic QWs. The decoherence process is originated from interface roughness, ${ }^{22}$ or inelastic scattering in the QW and at the interface, ${ }^{23}$ which can absorb and re-emit electrons losing their phase 
information. In addition, the small electron mean-free path in the metals (Fe: $1.5 \mathrm{~nm})^{24}$ also results in a rapid energy thermalization due to the electron-electron or electron-phonon scattering, which prevents electrons dwelling on the QW states before tunneling into a second QW. Those are the reason why elaborating efficient multi-QW structures is very challenging.

In our previous work, ${ }^{25}$ by using $\mathrm{MgAlO}_{x}$ as barrier $^{26-29}$ to form the $\mathrm{QW}$, we demonstrated the long-range phase coherence in thick Fe QW up to $12 \mathrm{~nm}$, which was attributed to the minimizing of interfacial strain at the $\mathrm{Fe} / \mathrm{MgAlO}_{x}$ interface due to the small lattice mismatch between $\mathrm{Fe}$ and

$\mathrm{MgAlO}_{x} \cdot{ }^{30,31}$ In this work, by taking advantage of the long-range phase coherence in $\mathrm{Fe} / \mathrm{MgAlO} x \mathrm{QW}$ structure, we have successfully fabricated fully epitaxial double-QW MTJs with three $\mathrm{MgAlO}_{x}$ tunnel barriers. The resonant tunneling through two QW states in a specifically designed double-QW structure has been evidenced by magneto-transport measurements. The understanding of the resonant tunneling mechanism in multi-QW MTJ provides efficient ways to engineer new functionalities for quantum resonant tunneling spintronic devices.

\section{Results and Discussion}

The single and double quantum-well (SQW and DQW) samples namely $\mathrm{MgO}$ substrate//MgO $(10 \mathrm{~nm}) / \mathrm{Fe}(45 \mathrm{~nm}) / \mathrm{SQW}$ or DQW/Fe $(10 \mathrm{~nm}) / \mathrm{Co}(10 \mathrm{~nm}) / \mathrm{Au}(10 \mathrm{~nm})$ were grown in a molecular beam epitaxy (MBE) chamber. Three types of DQW structures were designed in this study with the stack structures from bottom to top as following: Sample $I: \operatorname{MgAlO}_{x}(3 \mathrm{MLs}) / \mathrm{Fe}(9 \mathrm{~nm}) / \mathrm{MgAlO}_{x}(6$ MLs, etching stop layer $) / \mathrm{Fe}(9 \mathrm{~nm}) / \mathrm{MgAlO}_{x}(3 \mathrm{MLs})$, Sample II: $\mathrm{MgAlO}_{x}(6 \mathrm{MLs}$, etching stop layer $) / \mathrm{Fe}(9 \mathrm{~nm}) / \mathrm{MgAlO}_{x}(6 \mathrm{MLs}) / \mathrm{Fe}(7.7 \mathrm{~nm}) / \mathrm{MgAlO}_{x}(6 \mathrm{MLs})$ and Sample III: $\mathrm{MgAlO}_{x}(6 \mathrm{MLs}$, etching stop layer $) / \mathrm{Fe}(7.7 \mathrm{~nm}) / \mathrm{MgAlO}_{x}(3 \mathrm{MLs}) / \mathrm{Fe}(7.7 \mathrm{~nm}) / \mathrm{MgAlO}_{x}(6 \mathrm{MLs})$. One SQW sample (Sample IV) was grown to compare with DQW Sample III with the structure: $\operatorname{MgAlO}_{x}(6 \mathrm{MLs}) / \mathrm{Fe}(7.7$ 
$\mathrm{nm}) / \mathrm{MgAlO}_{x}(6 \mathrm{MLs}$, etching stop layer). Here, ML indicates atomic monolayer and the thickness of barrier was controlled by the high-energy electron diffraction (RHEED) intensity oscillation recorded during growth. The detailed condition can be found in Methods section and Supplementary S1. After elaboration, E-beam lithography was used to form the nanopillar junctions, with elliptical shape of $120 \times 240 \mathrm{~nm}^{2}$ (shown in the inset of Fig.1(a)). During the fabrication of nanopillar structure for SQW or DQW MTJ, the ion milling etching was stopped in a certain layer named stop layer. Please see in Supplementary S2 the effect of the position of the stop layer. For the two-terminal magneto-transport measurements, the negative bias voltage corresponds to the injection of electrons from top to bottom electrodes.

Scanning transmission electron microscope (STEM) characterizations was performed to verify the structure of the DQW MTJs. Fig. 1(a) and (d) show dark-field STEM images for Sample III in a large and magnified scale, respectively. In Fig. 1(a), one can observe sharp interfaces between $\mathrm{MgAlO}_{x}$ barriers (in black contrast) and Fe layers (in grey contrast). The QW and barrier layers appear quite flat and continuous, indicating a high quality of the triple-barrier MTJ structure. The magnifiedscale image further confirms that the two Fe QW layers have an identical thickness of 7.7nm. Energydispersive X-ray (EDS) spectroscopy has been performed to examine the chemical composition of the barriers. Fig. 1(b) and (c) show respectively the mapping of $\mathrm{Al}$ and $\mathrm{Mg}$ elements, indicating their homogenous distribution in the barriers. Fig. 1(e) displays the intensity profiles of $\mathrm{Mg}, \mathrm{Al}$ and $\mathrm{O}$ elements with the corresponding STEM image. From the ratio of different elements, the chemical composition is confirmed to be consistent in the three $\mathrm{MgAlO}_{x}$ barriers.

Demonstration of the formation of double QWs in one MTJ 
Our first goal is to demonstrate the successful realization of DQW states in one MTJ structure. To do that, one has to be able to grow the QW structure on relative thick tunneling barrier in order to measure a sizable tunneling resistance. In our previous work, ${ }^{25}$ the QW layer has been grown on the thin $3 \mathrm{ML}$ thick $\mathrm{MgAlO}_{x}$ or $\mathrm{MgO}$ bottom barriers. This thickness was too thin to obtain a reasonable junction resistance even with a nanopillar structure. The bottom barrier thickness should be further increased. Nevertheless, in that case the QW states are found to be attenuated rapidly when increasing the thickness of the bottom $\mathrm{MgAlO}_{x}$ layer (see Supplementary S3). This could be due to the increase of the surface roughness with the thickness of the oxide layer, resulting in the decoherence of electron phase at the $\mathrm{MgAlO}_{x} / \mathrm{Fe}$ interface. ${ }^{22}$ Fortunately, we have found that the $6 \mathrm{ML}$ thick $\mathrm{MgAlO}_{x}$ could be a good compromise to obtain both the QW states and a measurable resistance for the nanopillar junctions. Based on the aforementioned reasons, we have designed the triple-barrier MTJ (Sample I) to demonstrate the formation of the double QW states. The core structure comprises two thin barriers at both sides and one thick barrier in the middle separated by two Fe QW layers with identical thicknesses, as schematically shown in Fig. 2(a). In this structure, the resistance of the thin barrier is 100 times lower than that of the thick barrier, so that most of bias voltage drop occurs within the thick barrier. The QW energy levels of the bottom and top Fe QW layers are almost aligned with the Fermi levels $\left(E_{\mathrm{F}}\right)$ of the under and upper bulk Fe electrodes, respectively. As shown in Fig. 2(b), when the negative bias is applied, $E_{\mathrm{F}}$ of the upper Fe electrode and the top QW energy levels are lifted up together. When the energy of electrons matches with the discrete QW states in the bottom QW layer under a specific bias, it results in the resonant tunneling and a local maximum of conductance. Thus, the oscillations of the conductance under the negative bias reflects the QW states formed in the bottom Fe QW layer. In a similar way, the QW states within the top QW layer can be detected by applying a positive bias, as schematically shown in Fig. 2(c). If the QW states can be formed in both QW layers, 
we expect to observe symmetric conductivity oscillation with bipolar bias (both positive and negative applied bias).

Fig. 2(d)-(g) show the transport properties of Sample I. The magneto-response TMR- $H$ curves at room temperature (RT) and $15 \mathrm{~K}$ are shown in Fig. 2(f). The magnetic field $(H)$ was applied along the Fe[100] easy axis direction and the TMR ratio is calculated by $\left(R_{\mathrm{AP}}-R_{\mathrm{P}}\right) / R_{\mathrm{P}} \times 100 \%$, where $R_{\mathrm{AP}}$ and $R_{\mathrm{P}}$ are the resistances of the antiparallel (AP) and parallel (P) magnetization configurations of the two adjacent QW layers, respectively. Since the middle thick barrier mainly dominates the junction resistance, the TMR ratio is indeed determined by the P and AP states of the two QW layers. From the TMR curve, it is found that the full AP state is difficult to reach due to the similar coercivity of the two Fe QW layers. Nevertheless, we still get a TMR ratio of $51 \%$ at RT due to the dipolar coupling between the two Fe QW layers within the nanopillar structure. ${ }^{32}$ Remarkably, quite symmetric oscillations in the differential conductance are observed under negative and positive bias in both $\mathrm{P}$ and AP states, as shown in Fig. 2(d) and (e). The amplitude of oscillation is much stronger in P state than that in AP state, while the oscillation periodicity can be well matched in both magnetization configurations and the oscillation is still detectable up to RT. The observation of conductivity oscillation in the AP state can be understood due to the band folding effect in the spinel $\mathrm{MgAl}_{2} \mathrm{O}_{4}$ MTJ, resulting in the existence of $\Delta_{1}$ conductive channel in the minority spin states, as demonstrated by the ab-initio calculation. ${ }^{33}$ In Fig. $2(\mathrm{~g})$, the differential TMR (DTMR), calculated from the differential conductance defined as DTMR $=\left[(d I / d V)_{P}-(d I / d V)_{A P}\right] /(d I / d V)_{A P}$, shows clear oscillations with the same periodicity as those of the conductivity oscillations.

To prove that the oscillations originate from the formation of the QW states, we performed simulations using a phase accumulation model (PAM). ${ }^{34}$ The PAM describes the quantization condition for the existence of a QW state as: 


$$
2 k_{\perp} d-\Phi_{1}-\Phi_{2}-\Phi_{\mathrm{inf}}=2 \pi n
$$

where $k_{\perp}=\sqrt{2 m^{*}\left(E-E_{L}\right)} / \hbar$ is the crystal momentum wave vector in the direction perpendicular to the interface, $d$ indicates the Fe QW thickness, and $\Phi_{1}=\Phi_{2}=2 \sin ^{-1} \sqrt{\left(E-E_{L}\right) /\left(E_{U}-E_{L}\right)}-$ $\pi$ are the reflection phase shift at the two $\mathrm{Fe} / \mathrm{MgAlO}$ interfaces which confine the QW structure. $m^{*}$ is the effective mass of majority $\Delta_{1}$ electron in $\mathrm{Fe}$, and $E_{L}$ and $E_{U}$ are the energies of the lower and upper edges of the barrier band gap. Here, we set $m^{*}=1, E_{L}=-1.0 \mathrm{eV}$ and $E_{U}=3.9 \mathrm{eV}$ similar to Ref. [8]. The parameter $\Phi_{\text {inf }}$ taking into account the additional phase shift at interfaces is set to 0 for simplification. By injecting all parameters into Eq. (1), we obtain the following expression:

$$
n=1.63 d \sqrt{V+1}-0.637 \sin ^{-1} \sqrt{\frac{V+1}{4.9}}+1-n_{0}
$$

Where $n_{0}$ is the index of the first QW state close to $E_{\mathrm{F}}$. As displayed in the inset of Fig. 2(d), with $n_{0}=15$, the positions of the local conductance maximum under bipolar bias conditions can be well fitted with the PAM model with an identical QW thickness $d=9.0 \mathrm{~nm}$ for both QWs. This clearly confirms the formation of double QW states in the triple-barrier MTJ Sample I.

\section{Resonant tunneling in MTJ with QWs in series}

To study the resonant tunneling through multi-QW structures, we have to float the energy levels in the QW and decouple the energy alignment between the QW states and $E_{\mathrm{F}}$ of thick upper and bottom Fe electrodes. For this purpose, we have designed Sample II with three $\mathrm{MgAlO}_{x}$ barriers with identical thickness of 6MLs while the thickness of top and bottom QWs is kept different (9nm and 7.7nm, respectively), as schematically shown in Fig. 3(a). In this sample, the voltage is dropped almost equally on the three barriers. Fig. 3(d)-(g) show the transport properties of Sample II. The TMR- $H$ curves at RT and $15 \mathrm{~K}$ are shown in Fig. 3(f). The clear resistance plateaus in the low magnetic 
field range indicates the establishment of a full AP state in this sample, which could be due to the enhanced AP dipolar coupling not only between the two Fe QW layers but also between the Fe QW layers and the thick Fe electrodes after the nanopillar structure formation. As a consequence, we have measured a higher TMR ratio of $110 \%$ at $15 \mathrm{~K}$ and $85 \%$ at $\mathrm{RT}$.

Fig. 3(d) and (e) show the bias dependence of the differential conductance in P and AP states, respectively. Clear oscillations are found for both positive and negative biases, but the periodicity is different. Normally, one would expect that the tunneling of electrons could have been influenced by the two QWs' states in series and thus two sets of conductance oscillation under one polar bias could be observed when the injector's $E_{\mathrm{F}}$ is aligned with the QW states in the two wells. However, our results clearly show that only one set of oscillation corresponding to the states in one QW is present. From the periodicity of the oscillation, it is reasonable to suppose that the conductance oscillations at positive and negative bias are attributed to the top and bottom QW states, respectively, due to their different QW thickness. The possible explanation is that the electrons are rapidly dephased and thermalized $^{35,36}$ inside the first QW before they can resonantly tunnel into the second QW, as schematically drawn in Fig. 3(b) and (c). In semiconductor QW, hot electrons typically thermalize in a picosecond time scale, dissipating energy via various phonon-mediated relaxation pathways. ${ }^{36}$ In metallic QW, electron thermalization is even enhanced due to the smaller electron mean-free path compared to the semiconductor. Therefore, the electrons will quickly lose their phase and energy coherence if they are confined in a QW for a longer time and the reception side with another QW structure has no available resonant QW energy level under that bias. In the end, the MTJ with QWs in series only reveals the resonant tunneling characters of the last QW which the electrons tunnel through. 
To further confirm our supposition, we have performed the analysis of the periodicity with the PAM model. To correctly take into account the electron thermalization effect, we have added a factor of $F$ in Eq. (2):

$$
n=1.63 d \sqrt{V * F+1}-0.637 \sin ^{-1} \sqrt{\frac{V * F+1}{4.9}}+1-n_{0}
$$

This $F$ factor represents the true voltage drop on the QW to probe the unoccupied QW states. As shown in the inset of Fig. 3(d), with the factor $F=0.56$, the periodicity at positive and negative bias can be well fitted with the states in the $7.3 \mathrm{~nm}$ and $8.7 \mathrm{~nm}$ thick QWs, respectively, which well correspond to the top and bottom QWs. In the situation of a full thermalization down to $E_{\mathrm{F}}$ in the first QW, one should obtain a $F=1 / 3$ by taking into account the voltage drop on one barrier. On the contrary, if the electrons maintain their energy when tunneling into the second QW, $F$ factor should be equal to $2 / 3$ due to the voltage drop on the two barriers. Therefore, our fitted factor $F=0.56$ between $1 / 3$ and 2/3 indicates that the electrons are partially thermalized in the first QW before tunneling into the second QW. One remarkable feature in this kind of DQW MTJ is that with the design of different thickness for the two QWs, one can achieve different oscillation periodicities with bipolar bias, which could be of interests to generate new functionality with the multi-QW MTJs.

Fig. 3(g) displays the DTMR curves calculated from the different conductance in P and AP states. Although clear oscillations can be observed in both positive and negative bias, the amplitude of oscillation is smaller than that of Sample I. This can be understood by noticing that the influence of junction resistance in Sample II due to the QW resonant tunneling only occurs in one QW, while the total junction resistance should take into account three tunneling barriers. Therefore, the oscillation amplitude is reduced by a factor of 3 compared to Sample I.

\section{Evidence of coherent resonant tunneling through double QWs}


As mentioned above, to achieve the coherent resonant tunneling through two QWs, one must accelerate the tunneling of electrons through QWs and minimize their energy thermalization inside the QWs. To fulfill the conditions for such kind of coherent resonant tunneling, the discrete QW energy levels in the two QWs should be aligned under a certain bias. The following three aspects were considered to design such DQW structure. Firstly, the two QW layers must have an identical thickness so that their whole set of QW states can be matched with each other at zero bias to fulfill the resonant tunneling condition. Secondly, the QW states should be decoupled with the $E_{\mathrm{F}}$ of thick electrodes so that one can adjust the bias to probe different unoccupied QW states. Thirdly, to ensure the energy alignment between the two QWs' states, the voltage drop between the two QWs should be minimized. Based on these considerations, we have designed a DQW MTJ structure (Sample III) with two thick barriers (6MLs) at both sides and one thin barrier (3MLs) in the middle, as schematically shown in Fig. 4(a). The two QW layers have identical thickness of 7.7nm. The difference in resistance between the thick and thin barriers ( 100 times) ensures that the main voltage is dropped on the two thick barriers and very small bias is applied across the two QWs when performing bias dependence measurements. Fig. 4(a) schematically shows the diagrams of the resonant tunneling through two QWs under a negative bias.

Fig. 4(b) shows the bias dependence of the differential conductance in P state. Oscillations in conductance can be clearly observed in the bipolar bias condition. However, the amplitude of oscillation under negative bias is stronger than that under positive bias. This could reflect a certain asymmetric growth condition for the $\mathrm{MgAlO}_{x} / \mathrm{Fe}$ interface and the $\mathrm{Fe} / \mathrm{MgAlO}$ interface. ${ }^{14}$ To prove that the observed quantum oscillation is originated from the coherent resonant tunneling between the two QWs, we have fabricated one reference sample (Sample IV) with SQW structure $\left[\mathrm{MgAlO}_{x}(6\right.$ $\left.\mathrm{MLs}) / \mathrm{Fe}(7.7 \mathrm{~nm}) / \mathrm{MgAlO}_{x}(6 \mathrm{MLs})\right]$, which is schematically shown in Fig. 4(c). In this sample, since 
the etching is stopped in the top thick $\mathrm{MgAlO}_{x}$ barrier, the applied bias is totally dropped across the top barrier due to its dominated resistance in the junction. In comparison, in the DQW sample, only half of bias is applied across one thick barrier to probe the unoccupied QW states. Therefore, to quantitively compare the QW state positions for both samples, we have intentionally multiplied by a factor of 2 for the bias voltage of Sample IV. Fig. 4(d) shows the parallel conductance as a function of the converted bias voltage for Sample IV. Strong oscillations can be observed only for negative bias voltage. This is due to the fact that the Fermi level $E_{\mathrm{F}}$ of the bottom electrode cannot be lifted to probe the unoccupied QW states under the positive bias due to the small resistance of the bottom barrier with large micron size of area. Remarkably, the four main peaks (indicated with dash lines) at negative bias correspond perfectly to those of Sample III. The slight difference in position is enlarged for the QW states at larger bias, which is due to a smaller QW thickness in Sample IV [7.0 nm estimated by PAM simulation in the inset of $4(\mathrm{~d})$ ]. This gives a strong argument that although the DQW structure possesses double 7.7nm thick QWs, it really acts as the same behavior of a single 7.7nm thick QW. The QW state positions can be well fitted in the inset of Fig. 4(b). This also proves that the electrons can coherently resonantly tunnel through the two QWs without energy thermalization in one of the QWs. These results would not have been observed if the electrons had thermalized in the QWs. The situation would have been similar to what we have observed for the SQW reference sample under positive bias condition.

To enhance coherent resonant tunneling through the two QWs, two conditions are crucial. Indeed both the thin middle barrier and the available energy levels in the second QW must ensure the electrons rapid tunneling into the second QW states without too much time dwelling in the first QW. Another remarkable feature for such coherent resonant tunneling appears in the resonant peak width. As clearly seen in Fig. 4(b) and 4(d), the width of the oscillation peak for the DQW Sample III is 
about half of SQW Sample IV. Since the QW states act as an energy filter, double QW structure can enhance this energy selection effect for the tunneling electrons, which results in narrower oscillation peak width. This finding also reinforces our conclusion that the observed oscillation features in Sample III are originated from the coherent resonant tunneling through the double QWs. One could also expect that the DQW must have stronger oscillation amplitude than that of SQW. Indeed the QW states in Fe are formed by majority $\Delta_{1}$ electron band. ${ }^{4,8}$ The QW can select not only the energy of tunneling electrons but also the Bloch state symmetry feature linked to the band structures. Therefore, the DQW can effectively suppress the conduction channels from other electron bands than the majority $\Delta_{1}$ band, resulting in an enhanced oscillation amplitude. However, the oscillation amplitude could also be influenced by many other factors, such as QW thickness and the etching stop layer (see Supplementary S2). A slight change in the QW layer thickness and the different etching stop layer in the SQW sample could effectively increase the oscillation amplitude and make the direct comparison with the DQW sample difficult. Undoubtedly, future reduction of the QW thickness in the DQW structure should exponentially increase the oscillation amplitude.

It is well known from quantum theory that in the multi-QW system, when the middle barrier is sufficient thin, the two sets of QW states in the two wells can hybridize with each other and create new set of QW states. ${ }^{37}$ For a qualitative description of such physics in our DQW structure, we calculated the tunneling ratio of MTJ by using classic transfer matrix method on two DQW structures: Thin barrier/QW/Thick barrier/QW/Thin barrier and Thick barrier/QW/Thin barrier/QW/Thick barrier. Please see details of calculation in Supplementary S4. Fig. 5 displays the calculated energy dependence of the tunneling ratio $(T)$ in the two kinds of DQW junctions. Evidently, the DQW junctions with three tunnel barriers exhibit peak splitting effect due to the hybridization between the QW states in the two wells. In the Thin barrier/QW/Thick barrier/QW/Thin barrier junction (Fig. 
5(a)), the QW states weakly interact with each other and the splitting appears clearly at high bias voltage when the electron waves start overlaping through the barriers. On the contrary, in the Thick barrier/QW/Thin barrier/QW/Thick barrier junction (Fig. 5(b)), QW states strongly interact with each other and one QW state can be completely split into two states. Generally, the splitting of the satellite peaks can be controlled by the thickness of the central barrier. In our experiments (Fig. 4(c)), the splitting in the resonant peaks cannot be detected. This could be due to two reasons. Either the middle barrier could be still too thick so that the hybridization of QW states is too weak. Or, the energy distribution of tunneling electrons is too large and the splitting of peak is submerged by the overlap of two satellite QW states. In the future, the optimization of middle barrier thickness will allow us to observe such hybridization between two QWs.

\section{Conclusion}

In summary, the spin-dependent resonant tunneling properties have been studied in fully epitaxial $\mathrm{MgAlO}_{x}$ MTJ with double quantum well structures. Strong oscillations in conductance have been observed in the nanopillar structured DQW MTJ. It is found that the middle barrier plays a critical role on the resonant tunneling through the two QWs in series. When it is too thick, the electrons are partially thermalized in the first QW, consequently the periodicity of the conductance oscillations are only determined by the second QW. When the resonant tunneling condition is fulfilled, the DQW MTJ can produce strong conductance oscillations with much narrower peak width (about half) owing to the enhanced energy selection effect compared to the SQW MTJ. The realization of multiple QWs in single MTJ paves an efficient way to engineer quantum resonant tunneling to control the magnetoresistance with bias in addition to the conventional magnetization manipulation. 


\section{Methods}

\section{Sample preparation:}

The fully epitaxial MTJs with different configurations of $\mathrm{MgAlO}_{x}$ barriers were grown by MBE on $\mathrm{MgO}(001)$ substrate. Firstly, the substrate was annealed at $650^{\circ} \mathrm{C}$ for 60 mins and $10 \mathrm{~nm} \mathrm{MgO}$ seed layer was deposited at RT to prevent the carbon diffusion during subsequent annealing process. ${ }^{38}$ Then $45 \mathrm{~nm} \mathrm{Fe}$ was grown at RT as bottom electrode and annealed at $500^{\circ} \mathrm{C}$ for 30 mins to improve the crystalline quality and flatten the surface. Double QW structures of $\mathrm{MgAlO}_{x} / \mathrm{Fe} / \mathrm{MgAlO}_{x} / \mathrm{Fe} / \mathrm{MgAlO}_{x}$ with different thickness of barriers and wells were then deposited, where each Fe layer was annealed at $400^{\circ} \mathrm{C}$ for 20 mins. The layer-by-layer growth of the $\mathrm{MgAlO}_{x}$ barriers at RT was monitored by the oscillation of in-situ RHEED intensity. Finally, the samples were capped with $\mathrm{Fe}(10 \mathrm{~nm}) / \mathrm{Co}(20 \mathrm{~nm}) / \mathrm{Au}(10 \mathrm{~nm})$ as the top electrode, where only Fe layer was annealed at $400^{\circ} \mathrm{C}$ for 20 mins. The Co layer is used to enhance the coercivity of top Fe and the Au layer is used to prevent the sample from oxidation. The multilayers were patterned into nanopillar junctions with elliptical size of $120 \times 240 \mathrm{~nm}^{2}$ by E-beam lithography combined with Ar ion etching technique. The etching process was monitored by secondary ion mass spectrometry (SIMS) and can be precisely stopped in the desired $\mathrm{MgAlO}_{x}$ barrier.

\section{TEM characterization:}

Thin lamella TEM samples were prepared by Focused Ion Beam (FIB) lift up method (FEI-FIBHelios). Ex-situ microstructural characterization was performed with probe aberration-corrected STEM microscopes (FEI-Titan-Themis) operated at $300 \mathrm{kV}$ equipped with the super $\mathrm{X}$ energy dispersive X-ray spectrometer (EDS). 


\section{Acknowledgement}

This work was supported by the National Key Research and Development Program of China (Grant No. 2017YFA0206200), the National Natural Science Foundation of China (NSFC) (Grant No.11434014, 51620105004, 11674373, 51701203), the Strategic Priority Research Program (B) (Grant No. XDB07030200), the Key Research Program of Frontier Sciences (Grant No. QYZDJSSW-SLH016), and the International Partnership Program of the Chinese Academy of Sciences (CAS) (No.112111KYSB20170090). We also acknowledge the support by the joint French National Research Agency (ANR)-NSFC SISTER project (Grants No. ANR-11-IS10-0001 and No. NNSFC 61161130527), ENSEMBLE (Grants No. ANR-14-0028-01 and No. NNSFC 61411136001). We thank the ANR FEOrgSpin project (Grants No. ANR-18-CE24-0017-01) and the French PIA project “Lorraine Université d'Excellence” (No. ANR-15-IDEX-04-LUE). Experiments were performed using equipment from the platform TUBE-DAUM funded by FEDER (EU), ANR, the Region Lorraine and Grand Nancy. 


\section{References:}

(1) Tsu, R.; Esaki, L. Tunneling in a finite superlattice. Appl. Phys. Lett. 1973, 22, 562.

(2) Julliere, M. Tunneling between ferromagnetic films. Phys. Lett. 1975, 54A, 225-226.

(3) Moodera, J. S.; Kinder, L. R.; Wong, T. M.; Meservey, R. Large magnetoresistance at room temperature in ferromagnetic thin film tunnel junctions. Phys. Rev. Lett. 1995, 74, 3273-3276.

(4) Yuasa, S.; Nagahama, T.; Suzuki, Y. Spin-polarized resonant tunneling in magnetic tunnel junctions. Science 2002, 297, 234-237.

(5) Chshiev, M.; Stoeffler, D.; Vedyayev A.; Ounadjela, K. Magnetic Diode Effect in Double Barrier Junctions. Europhys. Lett. 2002, 58, 257.

(6) Kalitsov, A.; Coho, A.; Kioussis, N.; Vedyayev, A.; Chshiev M.; Granovsky, A. Impurity-induced tuning of quantum well states in spin-dependent resonant tunneling. Phys. Rev. Lett. 2004, 93, 046603.

(7) Lu, Z. Y.; Zhang, X. G.; Pantelides, S. T. Spin-dependent resonant tunneling through quantum-well states in magnetic metallic thin films. Phys. Rev. Lett. 2005, 94, 207210.

(8) Wang, Y.; Lu, Z. Y.; Zhang, X. G.; Han, X. F. First-principles theory of quantum well resonance in double barrier magnetic tunnel junctions. Phys. Rev. Lett. 2006, 97, 087210.

(9) Teixeira, J. M.; Costa, J. D.; Ventura, J.; Sousa, J. B.; Wisniowski, P.; Freitas, P. P. Observation of spindependent quantum well resonant tunneling in textured CoFeB layers. Appl. Phys. Lett. 2014, 104, 112414.

(10) Sheng, P.; Bonell, F.; Miwa, S.; Nakamura, T.; Shiota, Y.; Murakami, S.; Lam, D. D.; Yoshida, S.; Suzuki, $\mathrm{Y}$. Detailed analysis of spin-dependent quantum interference effects in magnetic tunnel junctions with $\mathrm{Fe}$ quantum wells. Appl. Phys. Lett. 2013, 102, 032406.

(11) Greullet, F.; Tiusan, C.; Montaigne, F.; Hehn, M.; Halley, D.; Bengone, O.; Bowen, M.; Weber, W. Evidence of a symmetry-dependent metallic barrier in fully epitaxial $\mathrm{MgO}$ based magnetic tunnel junctions. Phys. Rev. Lett. 2007, 99, 187202.

(12) Niizeki, T.; Tezuka, N.; Inomata, K. Enhanced tunnel magnetoresistance due to spin dependent quantum well resonance in specific symmetry states of an ultrathin ferromagnetic electrode. Phys. Rev. Lett. 2008, 100, 047207.

(13) Zeng, Z. M.; Han, X. F.; Zhan, W. S.; Wang, Y.; Zhang, Z.; Zhang, S. Oscillatory tunnel magnetoresistance in double barrier magnetic tunnel junctions. Phys. Rev. B 2005, 72, 054419.

(14) Nozaki, T.; Tezuka, N.; Inomata, K. Quantum oscillation of the tunneling conductance in fully epitaxial double barrier magnetic tunnel junctions. Phys. Rev. Lett. 2006, 96, 027208.

(15) Liu, R. S.; Yang, S. H.; Jiang, X.; Zhang, X.-G.; Rettner, C.; Gao, L.; Topuria, T.; Rice, P. M.; Zhang, W.; Canali, C. M.; Parkin, S. S. P. CoFe alloy as middle layer for strong spin dependent quantum well resonant tunneling in $\mathrm{MgO}$ double barrier magnetic tunnel junctions. Phys. Rev. B 2013, 87, 024411.

(16) Iovan, A.; Andersson, S.; Naidyuk, Y. G.; Vedyaev, A.; Dieny, B.; Korenivski, V. Spin diode based on $\mathrm{Fe} / \mathrm{MgO}$ double tunnel junction. Nano Lett. 2008, 8, 805-809.

(17) Esaki, L.; Chang, L. L.; Tsu, R. Resonant tunneling in semiconductor double barriers. Appl. Phys. Lett. 1974, 24, 593-595. 
(18) Brown, E. R.; Sollner, T. C. L. G.; Parker, C. D.; Goodhue, W. D.; Chen, C. L. Oscillations up to 420 $\mathrm{GHz}$ in GaAs/AlAs resonant tunneling diodes. Appl. Phys. Lett. 1989, 55, 1777-1779.

(19) Zory, P. S. Jr. Quantum Well Lasers 1-13; Academic Press, 1993.

(20) Esaki, L.; Chang, L. L. New transport phenomenon in a semiconductor "superlattice". Phys. Rev. Lett. 1974, 33, 495-498.

(21) Davies, R. A.; Kelly, M. J.; Kerr, T. M. Tunneling between two strongly coupled superlattices. Phys. Rev. Lett. 1985, 55, 1114-1116.

(22) Chshiev, M.; Stoeffler, D.; Vedyayev, A.; Ounadjela, K. Influence of quantum well states on transport properties of double barrier junctions, J. Magn. Magn. Mater. 2002, 240, 146.

(23) Jonsonand, M.; Grincwajg, A. Effect of inelastic scattering on resonant and sequential tunneling in double barrier heterostructures. Appl. Phys. Lett. 1987, 51, 1729.

(24) Gurney, B. A.; Speriosu, V. S.; Nozieres, J-P.; Lefakis, H.; Wilhoit, D. R.; Need, O. U. Direct Measurement of Spin-Dependent Conduction-Electron Mean Free Paths in Ferromagnetic Metals. Phys. Rev. Lett. 1993, 71, 4023-4026.

(25) Tao, B. S.; Yang, H. X.; Zuo, Y. L.; Devaux, X.; Lengaigne, G.; Hehn, M.; Lacour, D.; Andrieu, S.; Chshiev, M.; Hauet, T.; Montaigne, F.; Mangin, S.; Han, X. F.; Lu, Y. Long-range phase coherence in doublebarrier magnetic tunnel junctions with a large thick metallic quantum well. Phys. Rev. Lett. 2015, 115, 157204.

(26) Tao, B. S.; Li, D. L.; Yuan, Z. H.; Liu, H. F.; Ali, S. S.; Feng, J. F.; Wei, H. X.; Han, X. F.; Liu, Y.; Zhao, Y. G.; Zhang, Q.; Guo, Z. B.; Zhang, X. X. Perpendicular magnetic anisotropy in Ta|Co40Fe40B20|MgAl2O4 structures and perpendicular $\mathrm{CoFeB}|\mathrm{MgAl} 2 \mathrm{O} 4| \mathrm{CoFeB}$ magnetic tunnel junction. Appl. Phys. Lett. 2014, 105, 102407.

(27) Belmoubarik, M.; Sukegawa, H.; Ohkubo, T.; Mitani, S.; Hono, K. MgAl2O4(001) based magnetic tunnel junctions made by direct sputtering of a sintered spinel target. Appl. Phys. Lett. 2016, 108, 132404.

(28) Ikhtiar; Sukegawa, H.; Xu, X.; Belmoubarik, M.; Lee, H.; Kasai, S.; Hono, K. Giant tunnel magnetoresistance in polycrystalline magnetic tunnel junctions with highly textured $\mathrm{MgAl} 2 \mathrm{O} 4(001)$ based barriers. Appl. Phys. Lett. 2018, 112, 022408.

(29) Zhang, J.; Zhang, X. G.; Han, X. F. Spinel oxides: $\Delta 1$ spin-filter barrier for a class of magnetic tunnel junctions. Appl. Phys. Lett. 2012, 100, 222401.

(30) Sukegawa, H.; Xiu, H.; Ohkubo, T.; Furubayashi, T.; Niizeki, T.; Wang, W.; Kasai, S.; Mitani, S.; Inomata, K.; Hono, K. Tunnel magnetoresistance with improved bias voltage dependence in lattice-matched $\mathrm{Fe} / \mathrm{spinel}$ MgA12O4/Fe(001)MgAl2O4/Fe(001) junctions. Appl. Phys. Lett. 2010, 96, 212505.

(31) Sukegawa, H.; Miura, Y.; Muramoto, S.; Mitani, S.; Niizeki, T.; Ohkubo, T.; Abe, K.; Shirai, M.; Inomata, K.; Hono, K. Enhanced tunnel magnetoresistance in a spinel oxide barrier with cation-site disorder. Phys. Rev. B 2012, 86, 184401.

(32) Matsumoto, R.; Fukushima, A.; Yakushiji, K.; Yakata, S.; Nagahama, T.; Kubota, H.; Katayama, T.; Suzuki, Y.; Ando, K.; Yuasa, S.; Georges, B.; Cros, V.; Grollier, J.; Fert, A. Spin-torque-induced switching and precession in fully epitaxial Fe/MgO/Fe magnetic tunnel junctions. Phys. Rev. B 2009, 80, 174405.

(33) Miura, Y.; Muramoto, S.; Abe, K.; Shirai, M. First-principles study of tunneling magnetoresistance in $\mathrm{Fe} / \mathrm{MgAl} 2 \mathrm{O} 4 / \mathrm{Fe}(001)$ magnetic tunnel junctions. Phys. Rev. B, 2012, 86, 024426. 
(34) Smith, N. V.; Brookes, N. B.; Chang, Y.; Johnson, P. D. Quantum-well and tight-binding analyses of spin-polarized photoemission from $\mathrm{Ag} / \mathrm{Fe}(001)$ overlayers. Phys. Rev. B 1994, 49, 332-338.

(35) Lyon, S. A. Thermalization of hot carriers in quantum wells. Superlattices and Microstructures 1987, 3, 261.

(36) Esmaielpour, H.; Whiteside, V. R.; Piyathilaka, H. P.; Vijeyaragunathan, S.; Wang, B.; Adcock-Smith, E.; Roberts, K. P.; Mishima, T. D.; Santos, M. B.; Bristow, A. D.; Sellers, I. R. Enhanced hot electron lifetimes in quantum wells with inhibited phonon coupling. Scientific Reports 2018, 8, 12473.

(37) Horn, K.; Schefller, M. Electronic structure 618; Elsevier Science B. V.: The Netherlands, 2000.

(38) Lu, Y.; Yang, H.-X.; Tiusan, C.; Hehn, M.; Chshiev, M.; Duluard, A.; Kierren, B.; Lengaigne, G.; Lacour, D.; Bellouard, C.; Montaigne, F. Spin-orbit coupling effect by minority interface resonance states in singlecrystal magnetic tunnel junctions. Phys. Rev. B 2012, 86, 184420. 


\section{Figures:}
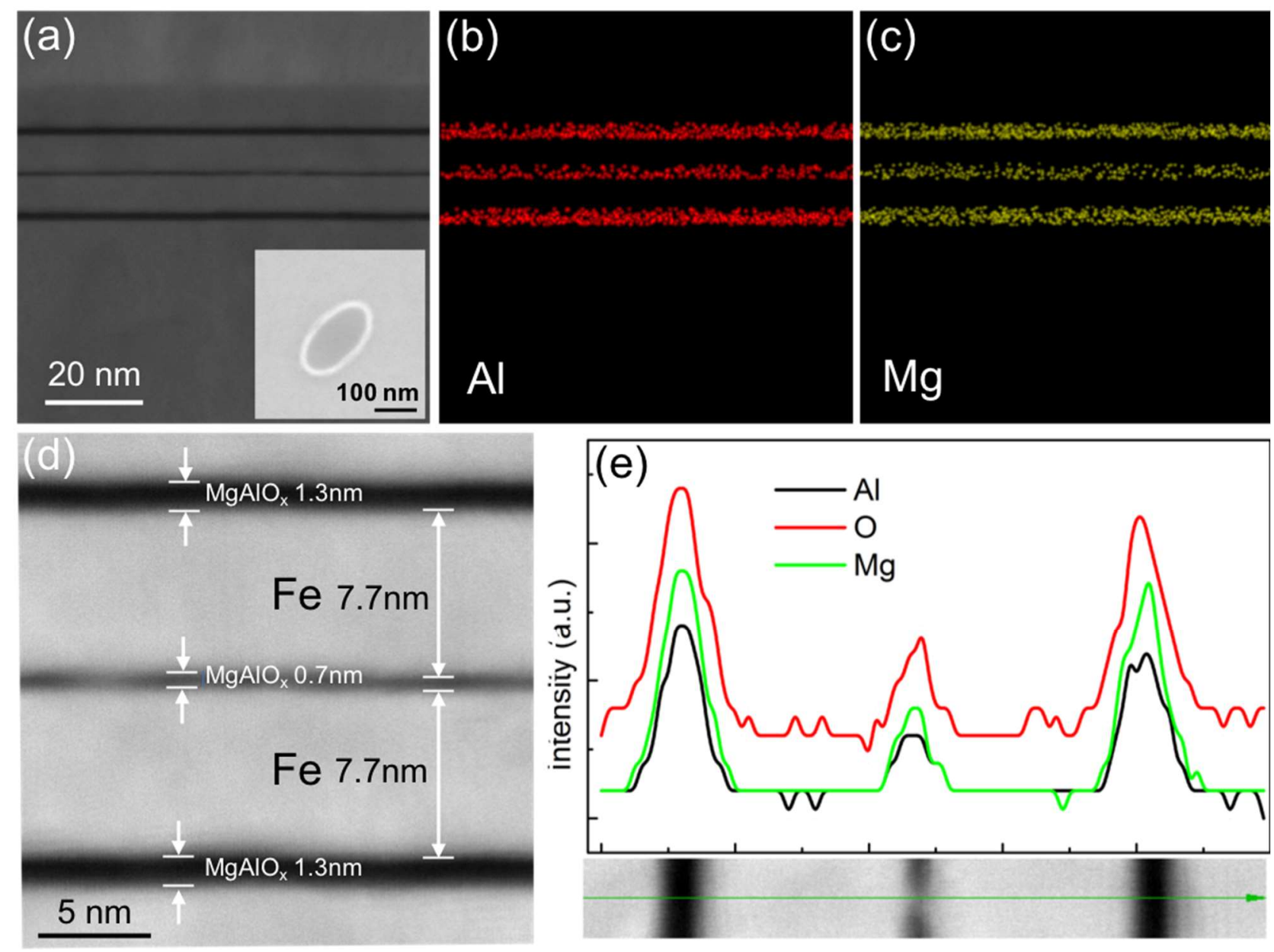

Figure 1: (a) Dark-field STEM image of the double QW MTJ. Inset: SEM top view of elliptical shape of nanopillar junction. (b) and (c) EDS mapping of Al and Mg element. (d) Enlarged STEM image in the double QW region. (e) Profile of different elements and corresponding STEM image. 
(a)

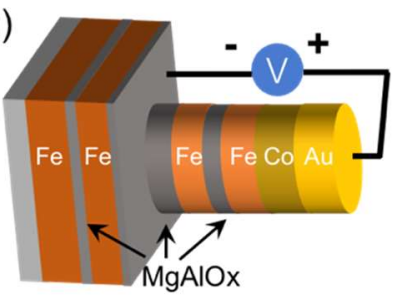

(b)

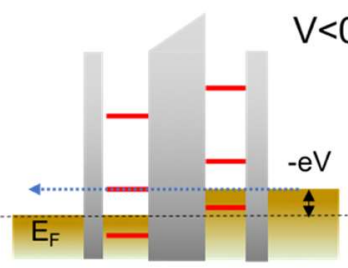

(c)

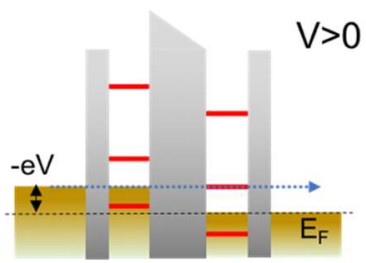

(d)
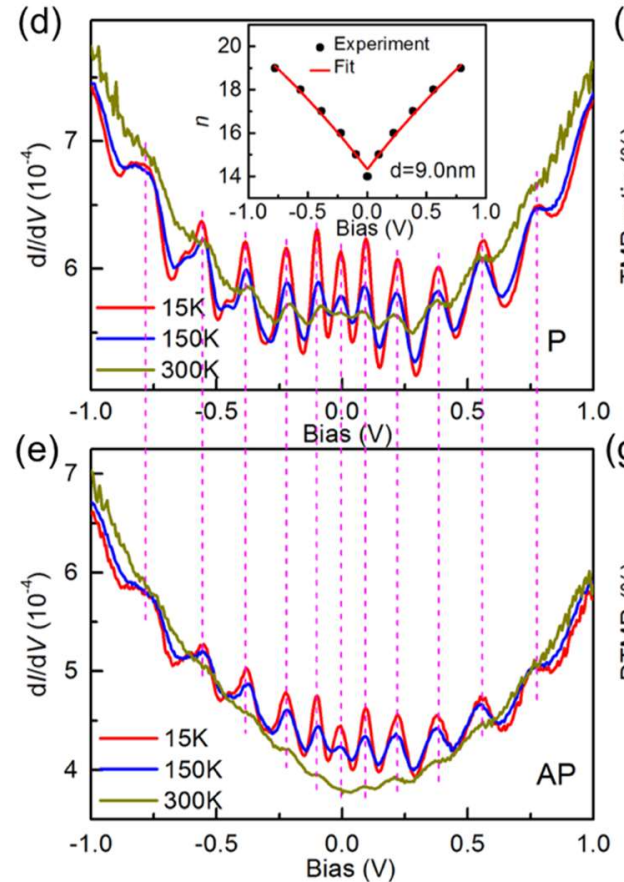
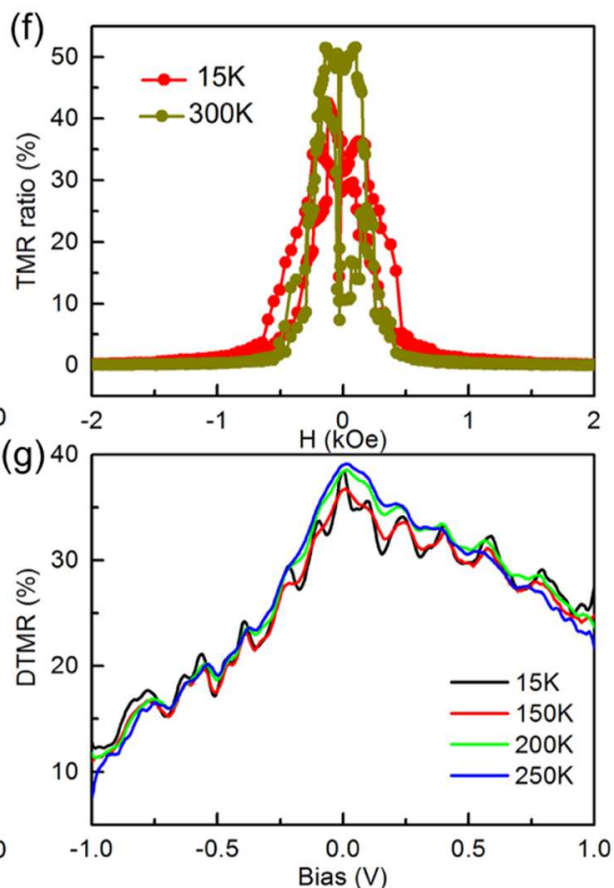

Figure 2: (a) Schematics of the double QW MTJ with core structure of $\mathrm{Fe} / \mathrm{MgAlO}_{x}(3$ $\mathrm{ML}) / \mathrm{Fe} / \mathrm{MgAlO}_{x}(6 \mathrm{ML}) / \mathrm{Fe} / \mathrm{MgAlO}_{x}(3 \mathrm{ML}) / \mathrm{Fe}$ and the setup of measurement. (b) and (c): Schematics of resonant tunneling in the sample with bias $\mathrm{V}<0$ and $\mathrm{V}>0$, respectively. (d) and (e): Bias dependence of differential conductance for the junction in magnetic parallel and antiparallel states, respectively. Inset in (d) shows the energy positions of QW states and the fitting by PAM method. (f) TMR-H curves of the junction at $T=15 \mathrm{~K}$ and $300 \mathrm{~K}$. (g) Bias dependence of differential TMR ratio at different temperatures. 
(a)

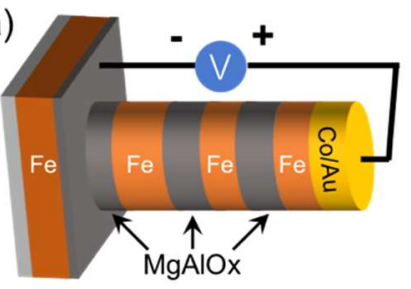

(b)

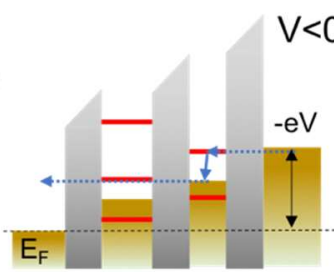

(c)

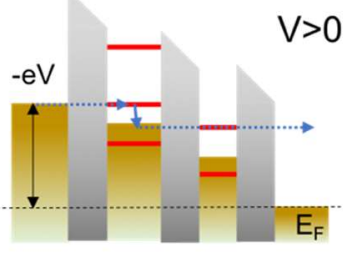

(d)
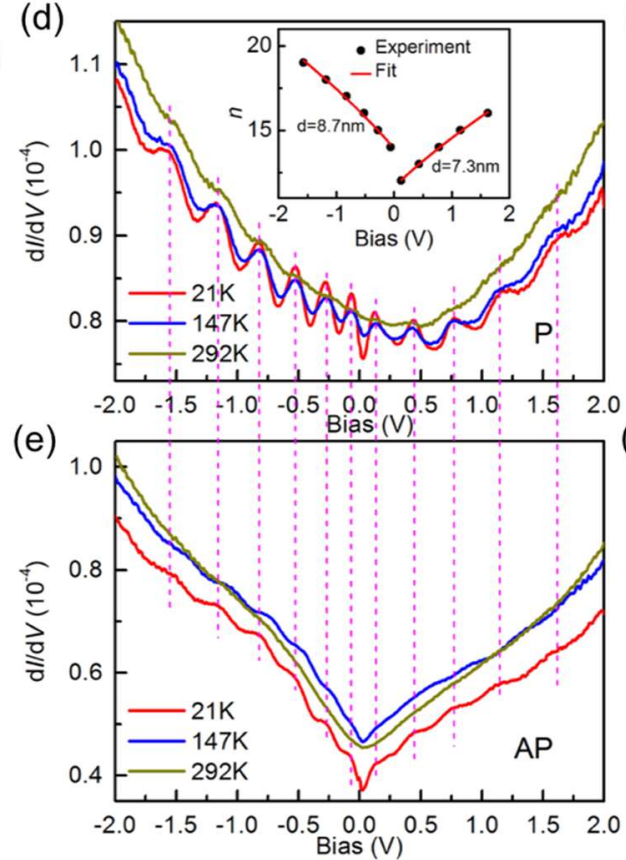

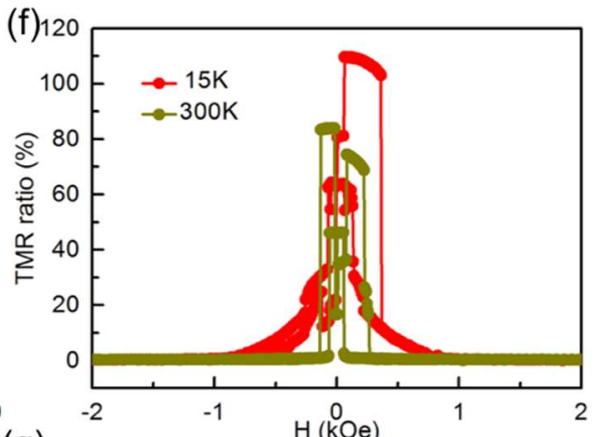

(g)

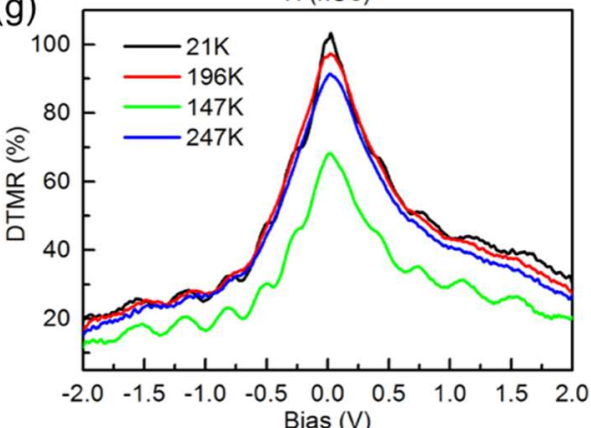

Figure 3: (a) Schematics of the double QW MTJ with core structure of $\mathrm{Fe} / \mathrm{MgAlO}_{x}(6$ $\mathrm{ML}) / \mathrm{Fe} / \mathrm{MgAlO}_{x}(6 \mathrm{ML}) / \mathrm{Fe} / \mathrm{MgAlO}_{x}(6 \mathrm{ML}) / \mathrm{Fe}$ and the setup of measurement. (b) and (c) Schematics of resonant tunneling in the sample with bias $\mathrm{V}<0$ and $\mathrm{V}>0$, respectively. The energy of electrons is partially thermalized before tunneling into the second QW. (d) and (e): Bias dependence of differential conductance for the junction in magnetic parallel and antiparallel states, respectively. Inset in (d) shows the energy positions of QW states and the fitting by PAM method. (f) TMR- $H$ curves of the junction at $T=15 \mathrm{~K}$ and $300 \mathrm{~K}$. (g) Bias dependence of differential TMR ratio at different temperatures. 
(a)

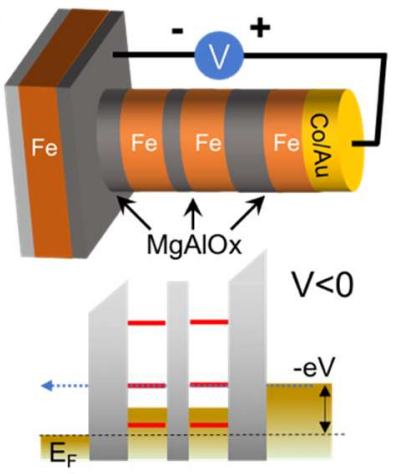

(c)
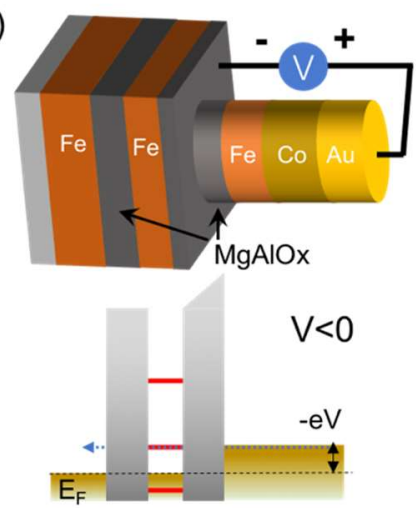
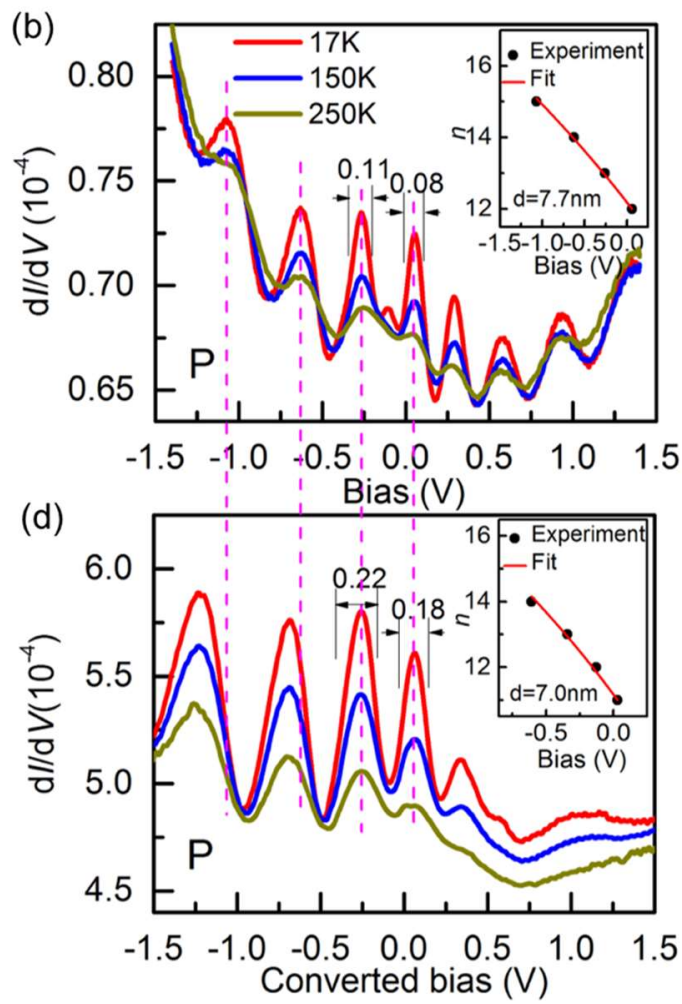

Figure 4: (a) Schematics of double QW MTJ with core structure of $\mathrm{Fe} / \mathrm{MgAlO}_{x}(6$ $\mathrm{ML}) / \mathrm{Fe} / \mathrm{MgAlO}_{x}(3 \mathrm{ML}) / \mathrm{Fe} / \mathrm{MgAlO}_{x}(6 \mathrm{ML}) / \mathrm{Fe}$ and schematics of the coherent resonant tunneling through double QWs with bias $\mathrm{V}<0$. (b) Bias dependence of differential conductance for the DQW junction represented in panel (a) in magnetic parallel state. (c) Schematics of single QW MTJ with core structure of $\mathrm{Fe} / \mathrm{MgAlO}_{x}(6 \mathrm{ML}) / \mathrm{Fe} / \mathrm{MgAlO}_{x}(6 \mathrm{ML}) / \mathrm{Fe}$ and schematics of the resonant tunneling in the junction with bias $\mathrm{V}<0$. (d) Bias dependence of differential conductance for the SQW junction shown in panel (c) in magnetic parallel state. The bias is multiplied by a factor of 2 in order to compare with panel (b). Please see details in main text. Insets of (b) and (d) show the energy positions of QW states and the fitting by PAM method. 

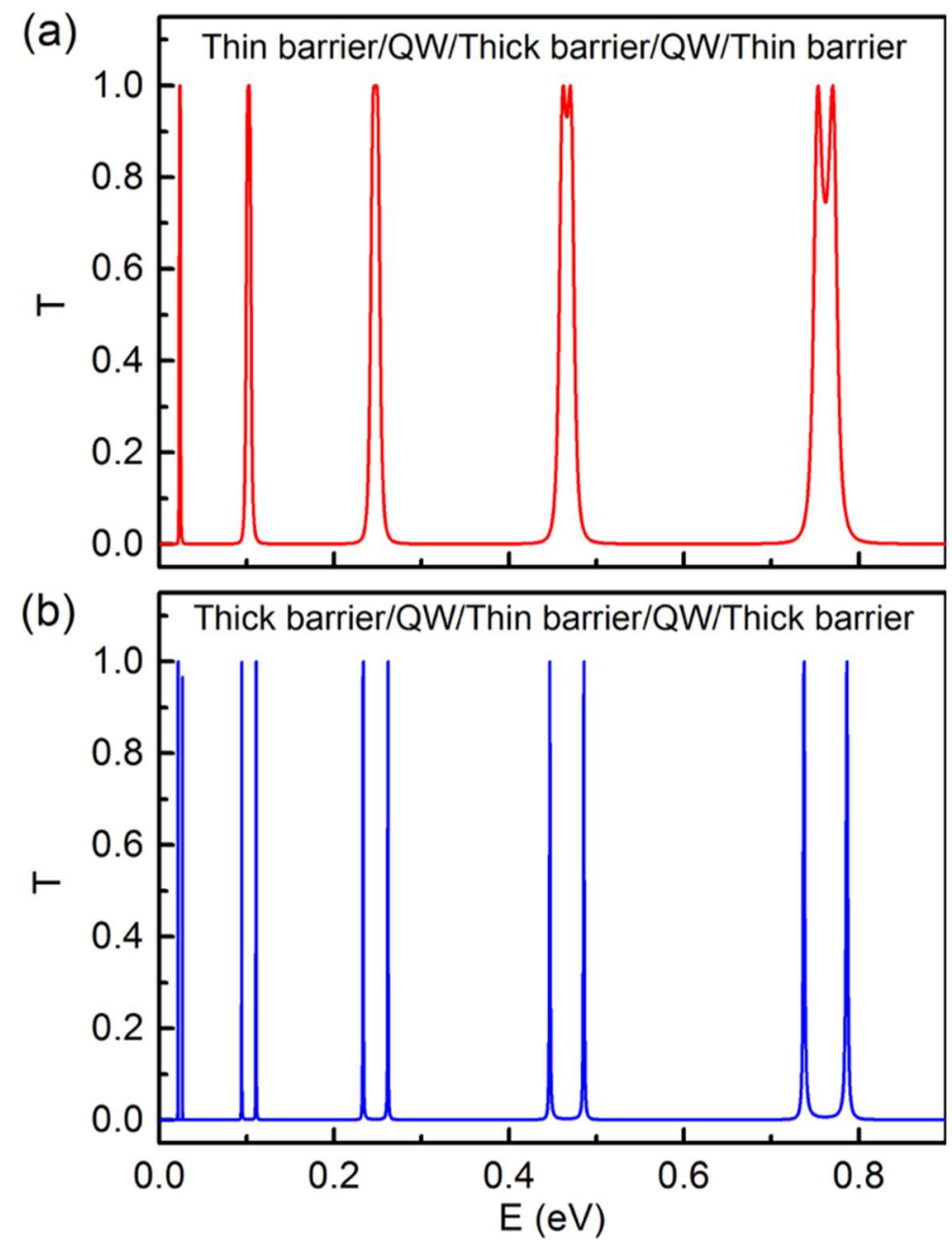

Figure 5: Simulation of energy dependence of tunneling ratio for double QW MTJs with structure of (a) Thick barrier/QW/Thin barrier/QW/Thick barrier and (b) Thin barrier/QW/Thick barrier/QW/Thin barrier, respectively. 


\section{TOC figure:}
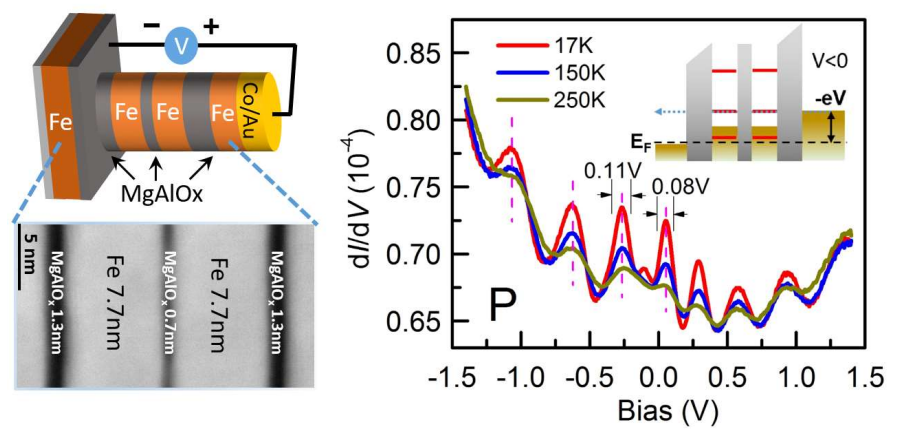

We demonstrate the successful fabrication of double quantum well (QW) structure in a single fully epitaxial magnetic tunnel junction heterostructure, where two Fe QW layers are sandwiched between three $\mathrm{MgAlO}_{x}$ tunnel barriers. We show clear evidence of the coherent resonant tunneling through the discrete QW states in the two QWs. 